\title{
Essential components of the set of solutions for the system of vector quasi-equilibrium problems
}

\author{
Xun-Hua Gong ${ }^{1 *}$ and Jian-Chen Chen ${ }^{2}$
}

\section{"Correspondence:}

xunhuagong@gmail.com

'Department of Mathematics,

Nanchang University, Nanchang,

330031, China

Full list of author information is

available at the end of the article

\begin{abstract}
In this paper, we prove that, for every vector quasi-equilibrium problem, there exists at least one essential component of the set of its solutions. As application, we show that, for every system of vector quasi-equilibrium problems, there exists at least one essential component of the set of its solutions in the uniform topological space of objective functions and constraint mappings.
\end{abstract}

\section{Introduction}

Essential component has been an important aspect in the study of stability for nonlinear problems. Fort [1] first introduced the notion of essential fixed points of a continuous mapping from a compact metric space into itself and proved that any mapping can be approximately closed by a mapping whose fixed points are all essential. Kinoshita [2] then introduced the notion of essential components of the set of fixed points of a single-valued map. Jiang [3] introduced the notion of essential components of the set of Nash equilibrium points for an n-person non-cooperative game and proved the existence of essential components of the set of Nash equilibrium points. Kohlberg and Mertens [4] studied the stability of Nash equilibrium points and suggested that a satisfactory solution for a noncooperative game should be set-wise, and they proved that such a solution is just an essential component of Nash equilibrium points. Recently, Yu, Xiang [5], Yu, Luo [6], Isac, Yuan [7], Yang, Yu [8], Lin [9], Chen, Gong [10] introduced the notion of essential components to solution sets of various problems such as Ky Fan point problems, equilibrium problems, coincident point problems, vector optimization problem, and symmetric vector quasi-equilibrium problems. On the other hand, in order to describe the real world and economic behavior better, very recently, much attention has been attracted to multicriteria equilibrium models. Ansari, Schaible and Yao [11] studied the system of generalized vector equilibrium problems. Ansari, Chan and Yang [12] studied the system of vector quasi-equilibrium problems (briefly, SVQEP). Fang, Huang and Kim [13] studied the system of vector equilibrium problems. Peng, Lee, Yang [14] studied the system of generalized vector quasi-equilibrium problems with set-valued maps (briefly, SGVOEPS). Lin [15] studied the system of generalized vector quasi-equilibrium problems (briefly, SGVQEP) in Banach spaces. Peng, Yang and Zhu [16] studied the system of vector quasi-equilibrium. Lin [9] established essential components of the solution set for SGVQEP under perturbations of the best-reply map. But up to now, no paper has established essential components

(c) 2012 Gong and Chen; licensee Springer. This is an Open Access article distributed under the terms of the Creative Commons Attribution License (http://creativecommons.org/licenses/by/2.0), which permits unrestricted use, distribution, and reproduction in any medium, provided the original work is properly cited. 
of the solution set for SVQEP, SGVQEP or SGVQEPS under perturbations of objective functions and constraint mappings. In this paper, we first give a new result of essential components of the solution set for SVQEP under perturbations of objective functions and constraint mappings.

\section{Preliminaries and definitions}

Let $I=\{1,2, \ldots, n\}$ be a finite set which has at least two elements. For each $i \in I$, let $X_{i}$ and $Y_{i}$ be real Hausdorff topological vector spaces and $K_{i}$ a nonempty subset of $X_{i}$. For each $i \in I$, let $C_{i}$ be a closed, convex and pointed cone of $Y_{i}$ with int $C_{i} \neq \emptyset$, where $\operatorname{int} C_{i}$ denotes the interior of $C_{i}$. Let $K=\prod_{i=1}^{n} K_{i}$. For each $i \in I$, let $f_{i}: K \times K_{i} \rightarrow Y_{i}$ be a vector-valued mapping and $S_{i}: K \rightarrow 2^{K_{i}}$ be a set-valued mapping. The SVQEP consists of finding $\bar{x} \in K$ such that for each $i \in I$,

$$
\bar{x}_{i} \in S_{i}(\bar{x}) \quad \text { and } \quad f_{i}\left(\bar{x}, y_{i}\right) \notin-\operatorname{int} C_{i} \quad \text { for all } y_{i} \in S_{i}(\bar{x})
$$

where $\bar{x}_{i}$ denotes the $i$ th component of $\bar{x}$, and $\bar{x}$ is said to be a solution of the SVQEP. For each $i \in I, f_{i}$ is said to be an objective function of the SVQEP and for each $i \in I, S_{i}$ is said to be a constraint mapping of the SVQEP. The SVQEP includes, as a special case, the following multiobjective generalized game problem:

For each $i \in I$, let $g_{i}: K \rightarrow Y_{i}$ be a vector-valued mapping and $G_{i}: K_{\hat{i}} \rightarrow 2^{K_{i}}$ be a feasible strategy mapping, where $K_{\hat{i}}=\prod_{j \in I, j \neq i} K_{j}$. For each $x \in K$, we can write $x=\left(x_{i}, x_{\hat{i}}\right)$. The multiobjective generalized game problem consists of finding $\bar{x} \in K$ such that for each $i \in I$, $\bar{x}_{i} \in G_{i}\left(\bar{x}_{i}\right)$ and

$$
g_{i}\left(y_{i}, \bar{x}_{\hat{i}}\right)-g_{i}\left(\bar{x}_{i}, \bar{x}_{\bar{i}}\right) \notin-\operatorname{int} C_{i} \quad \text { for all } y_{i} \in G_{i}\left(\bar{x}_{\bar{i}}\right)
$$

where $\bar{x}$ is said to be a weakly Pareto-Nash equilibrium point.

For each $i \in I$, setting

$$
f_{i}\left(x, y_{i}\right)=g_{i}\left(y_{i}, x_{\hat{i}}\right)-g_{i}\left(x_{i}, x_{\hat{i}}\right) \text { and } S_{i}(x)=G_{i}\left(x_{\hat{i}}\right)
$$

the SVQEP coincides with the multiobjective generalized game problem, which has been studied by Yu and Luo [6] but for real functions and Lin [9] but for $Y_{i}=R^{k_{i}}\left(1 \leq k_{i} \leq n\right)$ for any $i \in I$.

For each $i \in I$, setting $G_{i}\left(x_{i}\right)=K_{i}$, the multiobjective generalized game problem coincides with the multiobjective game problem, which has been studied by Yu and Xiang [5] and Yang and $\mathrm{Yu}[8]$.

Definition 2.1 Let $X$ be a real Hausdorff topological space and $Y$ a real Hausdorff topological vector space with a convex cone $C$. Let $f: X \rightarrow Y$ be a vector-valued function.

(i) $f$ is said to be $C$-continuous at $x_{0} \in X$ if, for any open neighborhood $V$ of the zero element $\theta$ in $Y$, there is an open neighborhood $N\left(x_{0}\right)$ of $x_{0}$ in $X$ such that

$$
f(x) \in f\left(x_{0}\right)+V+C \quad \text { for all } x \in N\left(x_{0}\right) .
$$

$f$ is said to be $C$-continuous on $X$ if it is $C$-continuous at every element of $X$. 
(ii) $f$ is said to be $(-C)$-continuous at $x_{0} \in X$ if, for any open neighborhood $V$ of $\theta$ in $Y$, there exists an open neighborhood $N\left(x_{0}\right)$ of $x_{0}$ in $X$ such that

$$
f(x) \in f\left(x_{0}\right)+V-C \text { for all } x \in N\left(x_{0}\right) .
$$

$f$ is said to be $(-C)$-continuous on $X$ if it is $(-C)$-continuous at every point of $X$.

Definition 2.2 Let $K$ be a nonempty convex subset of a vector space $X$, let $Y$ be a vector space with a convex pointed cone $C$. Let $f: K \rightarrow Y$ be a mapping. $f$ is said to be $C$-convex if, for any $x, y \in K$ and $t \in[0,1]$,

$$
t f(x)+(1-t) f(y)-f(t x+(1-t) y) \in C .
$$

Definition 2.3 Let $X$ and $Y$ be two Hausdorff topological spaces, let $F: X \rightarrow 2^{Y}$ be a setvalued mapping. $F$ is said to be upper semicontinuous (in short, u.s.c.) at $x_{0} \in X$ if, for any neighborhood $N\left(F\left(x_{0}\right)\right)$ of $F\left(x_{0}\right)$, there exists a neighborhood $N\left(x_{0}\right)$ of $x_{0}$ such that

$$
F(x) \subset N\left(F\left(x_{0}\right)\right) \text { for all } x \in N\left(x_{0}\right) \text {. }
$$

$F$ is said to be upper semicontinuous on $X$ if $F$ is u.s.c. at every point $x \in X$.

$F$ is said to be lower semicontinuous (in short, l.s.c.) at $x_{0} \in X$ if, for any $y_{0} \in F\left(x_{0}\right)$ and any neighborhood $N\left(y_{0}\right)$ of $y_{0}$, there exists a neighborhood $N\left(x_{0}\right)$ of $x_{0}$ such that

$$
F(x) \cap N\left(y_{0}\right) \neq \varnothing \quad \text { for all } x \in N\left(x_{0}\right) \text {. }
$$

$F$ is said to be lower semicontinuous on $X$ if it is lower semicontinuous at every $x \in X$.

$F$ is said to be continuous on $X$ if it is both u.s.c. and l.s.c. on $X$.

$F$ is said to be a closed mapping if Graph $F=\{(x, y) \in X \times Y: y \in F(x)\}$ is a closed set in $X \times Y$.

$F$ is an usco mapping if $F$ is u.s.c. on $X$ and $F(x)$ is compact for every $x \in X$.

Let $(X, d)$ be a linear metric space. Denote by $C K(X)$ all nonempty convex compact subsets of $X$. Define the Hausdorff metric $h$ on $C K(X)$ as follows.

For any $S_{1}, S_{2} \in C K(X)$, let

$$
h\left(S_{1}, S_{2}\right)=\max \left\{h^{\circ}\left(S_{1}, S_{2}\right), h^{\circ}\left(S_{2}, S_{1}\right)\right\},
$$

where

$$
h^{\circ}\left(S_{1}, S_{2}\right)=\sup \left\{d\left(b, S_{2}\right): b \in S_{1}\right\}
$$

and

$$
d\left(b, S_{2}\right)=\inf \left\{d(b, s): s \in S_{2}\right\} .
$$

Theorem 2.1 [17] Let $Y$ be a real Hausdorff topological vector space, and $C \subset Y$ be a closed convex pointed cone with int $C \neq \emptyset$. Let $K$ be a nonempty compact convex subset 
of a real locally convex Hausdorff topological vector space $X$. Let the set-valued mapping $S: K \rightarrow 2^{K}$ be continuous with nonempty compact convex values. If $\psi: K \times K \rightarrow Y$ satisfies the following conditions:

(i) $\psi(\cdot, \cdot)$ is $(-C)$-continuous;

(ii) for any fixed $x \in K, \psi(x, \cdot)$ is $C$-convex;

(iii) for any $x \in K, \psi(x, x) \notin-$ int $C$.

Then, there exists an element $x^{*} \in K$ such that $x^{*} \in S\left(x^{*}\right)$ and

$$
\psi\left(x^{*}, y\right) \notin-\operatorname{int} C \quad \text { for all } y \in S\left(x^{*}\right) .
$$

\section{Essential components of the solution set for the system of vector quasi-equilibrium problems}

Throughout this section, let $I=\{1,2, \ldots, n\}$ be a finite set which has at least two elements. For each $i \in I$, let $X_{i}$ be a real normed linear space and $Y_{i}$ a Banach space with $Y_{i} \subset Y_{n}$; let $K_{i}$ be a nonempty compact convex subset of $X_{i}$, and let $C_{i}$ be a closed convex pointed cone of $Y_{i}$ with $C_{i}=C_{n} \cap Y_{i}$ and int $C_{i} \neq \emptyset$. Let $K=\prod_{i=1}^{n} K_{i}$ and $X=\prod_{i=1}^{n} X_{i}$.

Let $\Phi$ be the collection of all vector-valued functions such that $\psi: K \times K \rightarrow Y_{n}$ such that: (i) $\psi(x, y)$ is $\left(-C_{n}\right)$-continuous on $K \times K$; (ii) for each fixed $x \in K, \psi(x, \cdot)$ is $C_{n}$-convex; (iii) for any $x \in K, \psi(x, x)=\theta$, where $\theta$ is the zero element of $Y_{n}$; (iv) $\sup _{(x, y) \in K \times K}\|\psi(x, y)\|<$ $+\infty$.

Let $M$ be the collection of all set-valued mappings $S: K \rightarrow 2^{K}$ such that: (i) for each $x \in K, S(x)$ is convex and closed; (ii) $S$ is continuous on $K$.

Let $H=\Phi \times M$. For any $u_{1}=\left(\psi^{\prime}, S^{\prime}\right), u_{2}=\left(\psi^{\prime \prime}, S^{\prime \prime}\right) \in H$, define

$$
\rho_{1}\left(u_{1}, u_{2}\right)=\sup _{(x, y) \in K \times K}\left\|\psi^{\prime}(x, y)-\psi^{\prime \prime}(x, y)\right\|+\sup _{x \in K} h\left(S^{\prime}(x)-S^{\prime \prime}(x)\right),
$$

where $\|\cdot\|$ is the norm on $Y_{n}$ and $h$ is the Hausdorff metric defined on $C K(X)$. Clearly, $\left(H, \rho_{1}\right)$ is a metric space.

For any $u=(\psi, S) \in H$, by Theorem 2.1 , there exists a solution $x^{*} \in K$ to the vector quasiequilibrium problem: $x^{*} \in S\left(x^{*}\right)$ and

$$
\psi\left(x^{*}, y\right) \notin-\operatorname{int} C_{n} \quad \text { for all } y \in S\left(x^{*}\right) .
$$

For each $u=(\psi, S) \in H$, define

$$
F(u)=\left\{x \in K: x \in S(x) \text { and } \psi(x, y) \notin-\operatorname{int} C_{n} \text { for all } y \in S(x)\right\} .
$$

Thus $F(u) \neq \emptyset$ for any $u \in H$ and $u \mapsto F(u)$ indeed defines a set-valued mapping from $H$ to $K$.

The following lemma can be found in [18].

Lemma 3.1 Let $X$ be a metric space and $K(X)$ be the family of all nonempty compact subsets of $X$. Let $A, A_{n} \in K(X)(n=1,2, \ldots)$ satisfy the condition that for each open set $O$ containing $A$, there exists an integer $N$ such that whenever $n>N$, we have $A_{n} \subset O$. Then for any sequence $\left\{x_{n}\right\}$ with $x_{n} \in A_{n}(n=1,2, \ldots)$, there exists a subsequence which converges to a point in $A$. 
Lemma 3.2 $F: H \rightarrow 2^{K}$ is an usco mapping.

Proof Since $K$ is compact, by [19], it suffices to prove that $\operatorname{Graph}(F)=\{(u, x) \in H \times K: x \in$ $F(u)\}$ is closed. Let $\left\{\left(u_{n}, x_{n}\right)\right\} \subset \operatorname{Graph}(F)$ with $\left(u_{n}, x_{n}\right) \rightarrow(u, \bar{x}) \in H \times K$, where $u_{n}=\left(\psi_{n}, S_{n}\right)$ and $u=(\psi, S)$. Since $x_{n} \in F\left(u_{n}\right)$, we have

$$
x_{n} \in S_{n}\left(x_{n}\right) \quad \text { and } \quad \psi_{n}\left(x_{n}, y\right) \notin-\operatorname{int} C_{n} \quad \text { for all } y \in S_{n}\left(x_{n}\right) .
$$

For any open neighborhood $O$ of $S(\bar{x})$ in $K$, since $S(\bar{x})$ is compact, by [19, p.108], there is $\varepsilon_{0}>0$ such that

$$
\left\{x \in K: d(x, S(\bar{x}))<\varepsilon_{0}\right\} \subset O,
$$

where $d(x, S(\bar{x}))=\inf _{a \in S(\bar{x})}\|x-a\|$. Since $\rho_{1}\left(\left(\psi_{n}, S_{n}\right),(\psi, S)\right) \rightarrow 0, x_{n} \rightarrow \bar{x}$, and $S$ is u.s.c. at $\bar{x}$, there is $N$ such that for any $n>N$, we have

$$
\sup _{x \in K} h\left(S_{n}(x), S(x)\right)<\varepsilon_{0} / 2,
$$

and

$$
S\left(x_{n}\right) \subset\left\{x \in K: d(x, S(\bar{x}))<\varepsilon_{0} / 2\right\} .
$$

So whenever $n>N$, we have

$$
S_{n}\left(x_{n}\right) \subset\left\{x \in K: d\left(x, S\left(x_{n}\right)\right)<\varepsilon_{0} / 2\right\} \subset\left\{x \in K: d(x, S(\bar{x}))<\varepsilon_{0}\right\} \subset O .
$$

Since $x_{n}$ belongs to $S_{n}\left(x_{n}\right)$, and $S(\bar{x})$ and $S_{n}\left(x_{n}\right)$ are compact, by Lemma 3.1, there exists a subsequence $\left\{x_{n_{k}}\right\}$ of $\left\{x_{n}\right\}$ such that $x_{n_{k}} \rightarrow x_{0} \in S(\bar{x})$. Since $x_{n_{k}} \rightarrow \bar{x}$, we have

$$
\bar{x}=x_{0} \in S(\bar{x}) .
$$

Since $S$ is l.s.c. at $\bar{x} \in K$, for any $z \in S(\bar{x})$, by [19], there exists $a_{n} \in S\left(x_{n}\right)$ such that $a_{n} \rightarrow z$. Since $\rho_{1}\left(\left(\psi_{n}, S_{n}\right),(\psi, S)\right) \rightarrow 0$, there exists a subsequence $\left\{S_{n_{k}}\right\}$ of $\left\{S_{n}\right\}$ such that

$$
\sup _{x \in K} h\left(S_{n_{k}}(x), S(x)\right)<1 / k
$$

Thus, there exists a subsequence $\left\{x_{n_{k}}\right\}$ of $\left\{x_{n}\right\}$ such that

$$
h\left(S_{n_{k}}\left(x_{n_{k}}\right), S\left(x_{n_{k}}\right)\right)<1 / k
$$

which implies that there exists $a_{n_{k}}^{\prime} \in S_{n_{k}}\left(x_{n_{k}}\right)$ such that

$$
\left\|a_{n_{k}}^{\prime}-a_{n_{k}}\right\|<1 / k
$$

where $\left\{a_{n_{k}}\right\}$ is a subsequence of $\left\{a_{n}\right\}$. Since $\left\|a_{n_{k}}^{\prime}-z\right\| \leq\left\|a_{n_{k}}^{\prime}-a_{n_{k}}\right\|+\left\|a_{n_{k}}-z\right\|<1 / k+$ $\left\|a_{n_{k}}-z\right\|$ and $a_{n_{k}} \rightarrow z(k \rightarrow+\infty)$, we have that $a_{n_{k}}^{\prime} \rightarrow z(k \rightarrow+\infty)$. As $a_{n_{k}}^{\prime} \in S_{n_{k}}\left(x_{n_{k}}\right)$, we 
have

$$
\psi_{n_{k}}\left(x_{n_{k}}, a_{n_{k}}^{\prime}\right) \notin-\operatorname{int} C_{n} \quad \text { for all } k \text {. }
$$

Now we need to show that

$$
\psi(\bar{x}, z) \notin-\operatorname{int} C_{n} .
$$

If the conclusion is false, then $\psi(\bar{x}, z) \in-\operatorname{int} C_{n}$, which implies that there is $\bar{\varepsilon}>0$ such that

$$
\psi(\bar{x}, z)+\bar{\varepsilon} B \subset-\operatorname{int} C_{n},
$$

where $B$ denotes the open unit ball in $Y_{n}$. Since $\psi$ is $\left(-C_{n}\right)$-continuous on $K \times K, x_{n_{k}} \rightarrow \bar{x}$ and $a_{n_{k}}^{\prime} \rightarrow z$, for above $\bar{\varepsilon}>0$, there is a positive integer $k_{0}$ such that

$$
\psi\left(x_{n_{k}}, a_{n_{k}}^{\prime}\right) \in \psi(\bar{x}, z)+(1 / 2) \bar{\varepsilon} B-C_{n} \quad \text { for all } k \geq k_{0} .
$$

On the other hand, since $\rho_{1}\left(\left(\psi_{n_{k}}, S_{n_{k}}\right),(\psi, S)\right) \rightarrow 0$, there is a positive integer $k_{1}$ with $k_{1} \geq$ $k_{0}$, such that

$$
\psi_{n_{k}}(x, y) \in \psi(x, y)+(1 / 2) \bar{\varepsilon} B \quad \text { for any }(x, y) \in K \times K \text { and all } k \geq k_{1} \text {. }
$$

By (7), (6) and (5), we have

$$
\begin{aligned}
\psi_{n_{k}}\left(x_{n_{k}}, a_{n_{k}}^{\prime}\right) & \in \psi\left(x_{n_{k}}, a_{n_{k}}^{\prime}\right)+(1 / 2) \bar{\varepsilon} B \subset \psi(\bar{x}, z)+(1 / 2) \bar{\varepsilon} B+(1 / 2) \bar{\varepsilon} B-C_{n} \\
& \subset \psi(\bar{x}, z)+\bar{\varepsilon} B-C_{n} \subset-\operatorname{int} C_{n}-C_{n} \subset-\operatorname{int} C_{n} \quad \text { for all } k \geq k_{1} .
\end{aligned}
$$

This contradicts (3). Hence (4) holds. Then by the arbitrariness of $z \in S(\bar{x})$, we obtain that

$$
\psi(\bar{x}, z) \notin-\operatorname{int} C_{n} \quad \text { for all } z \in S(\bar{x}) .
$$

By (2) and (8), we have that $((\psi, S), \bar{x}) \in \operatorname{Graph}(F)$. Hence, $\operatorname{Graph}(F)$ is closed. $F(u)$ is also closed, for all $u \in H$. By the compactness of $K$, we know that $F$ is a set-valued mapping with compact values. Hence, $F$ is an usco mapping. The proof is completed.

For each $u \in H$, the component of a point $x \in F(u)$ is the union of all the connected subsets of $F(u)$ containing $x$. Note that the components are connected closed subsets of $F(u)$, and thus are connected and compact, see [20]. It is easy to see that the components of two distinct points of $F(u)$ either coincide or are disjoint, so that all components constitute a decomposition of $F(u)$ into connected pairwise disjoint compact subsets, i.e.,

$$
F(u)=\bigcup_{\alpha \in \Lambda} F_{\alpha}(u),
$$

where $\Lambda$ is an index set for each $\alpha \in \Lambda, F_{\alpha}(u)$ is a nonempty connected compact subset of $F(u)$ and, for any $\alpha, \beta \in \Lambda(\alpha \neq \beta), F_{\alpha}(u) \cap F_{\beta}(u)=\emptyset$. 
Definition 3.1 Let $u \in H$ and $m$ be a nonempty closed subset of $F(u) . m$ is said to be an essential set of $F(u)$ if, for each open set $O \supset m$, there exists $\delta>0$ such that for any $u^{\prime} \in H$ with $\rho_{1}\left(u, u^{\prime}\right)<\delta, F\left(u^{\prime}\right) \cap O \neq \emptyset$. If a component $F_{\alpha}(u)$ of $F(u)$ is an essential set, then $F_{\alpha}(u)$ is said to be an essential component of $F(u)$. An essential set $m$ of $F(u)$ is said to be a minimal essential set of $F(u)$ if $m$ is a minimal element of the family of essential sets in $F(u)$ ordered by set inclusion.

Lemma 3.3 [7] Let $A, B$ and $C$ be nonempty convex compact subsets of a normed linear space $X$. Then $h(A, \lambda B+\mu C) \leq \lambda h(A, B)+\mu h(A, C)$, where $h$ is the Hausdorff metric defined on $C K(X), \lambda \geq 0, \mu \geq 0$, and $\lambda+\mu=1$.

The following theorem is the exist theorem of an essential component of the set of solutions for the vector quasi-equilibrium problem.

\section{Theorem 3.1}

(a) For any $u \in H$, there exists at least one minimal essential set of $F(u)$, and every minimal essential set of $F(u)$ is connected;

(b) For any $u \in H$, there exists at least one essential connected component of $F(u)$.

\section{Proof}

(a) Since $F$ is upper semicontinuous, following the idea of Lemma 2.2 in [5], we can easily obtain that there exists one minimal essential set of $F(u)$ for each $u \in H$. Now, for each minimal essential set of $F(u)$, as Yang and Yu did in [8], we prove that each minimal essential set of $F(u)$ is connected. Let $m(u)$ be one minimal essential set of $F(u)$. If $m(u)$ is not connected, then there exist two nonempty closed sets $c_{1}(u)$ and $c_{2}(u)$ of $F(u)$ and two open sets $V_{1}$ and $V_{2}$ in $K$ such that

$$
m(u)=c_{1}(u) \cup c_{2}(u), \quad c_{1}(u) \subset V_{1}, \quad c(u) \subset V_{2}, \quad V_{1} \cap V_{2}=\emptyset .
$$

Since $m(u)$ is a minimal essential set of $F(u)$, neither $c_{1}(u)$ nor $c_{2}(u)$ is essential. Thus, there exist two open sets $O_{1} \supset c_{1}(u)$ and $O_{2} \supset c_{2}(u)$ such that, for any $\delta>0$, there exist $u_{1}, u_{2} \in H$ with

$$
\rho_{1}\left(u, u_{1}\right)<\delta, \quad \rho_{1}\left(u, u_{2}\right)<\delta, \quad \text { but } \quad F\left(u_{1}\right) \cap O_{1}=\emptyset, \quad F\left(u_{2}\right) \cap O_{2}=\emptyset .
$$

Set $W_{1}=V_{1} \cap O_{1}$, and $W_{2}=V_{2} \cap O_{2}$. Then both $W_{1}$ and $W_{2}$ are open sets and $c_{1}(u) \subset W_{1}$, and $c_{2}(u) \subset W_{2}$. Since $c_{1}(u)$ and $c_{2}(u)$ are a closed subset of the compact set $F(u), c_{1}(u)$ and $c_{2}(u)$ are a compact set, there exist two open sets $U_{1}$ and $U_{2}$ such that

$$
c_{1}(u) \subset U_{1} \subset \overline{U_{1}} \subset W_{1}, \quad c_{2}(u) \subset U_{2} \subset \overline{U_{2}} \subset W_{2}, \quad \text { and } \quad W_{1} \cap W_{2}=\emptyset .
$$

Since $m(u)$ is essential and $m(u) \subset U_{1} \cup U_{2}$, there exists $\delta^{\prime}>0$ such that for any $u^{\prime}$ with $\rho_{1}\left(u, u^{\prime}\right)<\delta^{\prime}$, we have

$$
F\left(u^{\prime}\right) \cap\left(U_{1} \cup U_{2}\right) \neq \emptyset .
$$


Since $U_{1} \subset O_{1}$ and $U_{2} \subset O_{2}$, for above $\delta^{\prime} / 2>0$, by (9), there exist $u_{1}, u_{2} \in H$ such that

$$
\rho_{1}\left(u, u_{1}\right)<\delta^{\prime} / 2, \quad \rho_{1}\left(u, u_{2}\right)<\delta^{\prime} / 2, \quad F\left(u_{1}\right) \cap U_{1}=\emptyset, \quad F\left(u_{2}\right) \cap U_{2}=\emptyset .
$$

Since $u_{1}, u_{2} \in H$, we have $u_{1}=\left(\psi_{1}, S_{1}\right)$ and $u_{2}=\left(\psi_{2}, S_{2}\right)$. Now we define $S^{\prime}: K \rightarrow 2^{K}$ and $\psi^{\prime}: K \times K \rightarrow Y_{n}$ by

$$
S^{\prime}(x)=\lambda(x) S_{1}(x)+\mu(x) S_{2}(x) \quad \text { for all } x \in K
$$

and

$$
\psi^{\prime}(x, y)=\lambda(x) \psi_{1}(x, y)+\mu(x) \psi_{2}(x, y) \quad \text { for all }(x, y) \in K \times K,
$$

respectively, where

$$
\lambda(x)=\frac{d\left(x, \overline{U_{2}}\right)}{d\left(x, \overline{U_{1}}\right)+d\left(x, \overline{U_{2}}\right)}, \quad \mu(x)=\frac{d\left(x, \overline{U_{1}}\right)}{d\left(x, \overline{U_{1}}\right)+d\left(x, \overline{U_{2}}\right)} \quad \text { for all } x \in K .
$$

It is obvious that $\lambda$ and $\mu$ are continuous functions on $K$ with $\lambda(x) \geq 0, \mu(x) \geq 0$, and $\lambda(x)+\mu(x)=1$ for any $x \in K$.

We can see that: (i) $\psi^{\prime}(x, y)$ is $\left(-C_{n}\right)$-continuous on $K \times K$; (ii) for each fixed $x \in K, \quad \psi^{\prime}(x, y)$ is $C_{n}$-convex in $y$; (iii) $\psi^{\prime}(x, x)=\theta \notin-\operatorname{int} C_{n}$ for all $x \in K$; (iv) $\sup _{(x, y) \in K \times K}\left\|\psi^{\prime}(x, y)\right\|<+\infty$; (v) for each $x \in K, S^{\prime}(x)$ is convex and compact; (vi) $S^{\prime}$ is continuous on $K$. Hence $v:=\left(\psi^{\prime}, S^{\prime}\right) \in H$. By Lemma 3.3, we have

$$
\begin{aligned}
h\left(S(x), S^{\prime}(x)\right) & \leq \lambda(x) h\left(S(x), S_{1}(x)\right)+\mu(x) h\left(S(x), S_{2}(x)\right) \\
& \leq h\left(S(x), S_{1}(x)\right)+h\left(S(x), S_{2}(x)\right) \quad \text { for all } x \in K,
\end{aligned}
$$

and

$$
\begin{aligned}
\left\|\psi(x, y)-\psi^{\prime}(x, y)\right\|= & \left\|\psi(x, y)-\lambda(x) \psi_{1}(x, y)-\mu(x) \psi_{1}(x, y)\right\| \\
\leq & \lambda(x)\left\|\psi(x, y)-\psi_{1}(x, y)\right\|+\mu(x)\left\|\psi(x, y)-\psi_{2}(x, y)\right\| \\
\leq & \left\|\psi(x, y)-\psi_{1}(x, y)\right\| \\
& +\left\|\psi(x, y)-\psi_{2}(x, y)\right\| \quad \text { for all }(x, y) \in K \times K .
\end{aligned}
$$

Thus, by (11), we have

$$
\begin{aligned}
\rho_{1}(u, v)= & \sup _{(x, y) \in K \times K}\left\|\psi(x, y)-\psi^{\prime}(x, y)\right\|+\sup _{x \in K} h\left(S(x), S^{\prime}(x)\right) \\
\leq & \sup _{(x, y) \in K \times K}\left\|\psi(x, y)-\psi_{1}(x, y)\right\|+\sup _{(x, y) \in K \times K}\left\|\psi(x, y)-\psi_{2}(x, y)\right\| \\
& \quad+\sup _{x \in K} h\left(S(x), S_{1}(x)\right)+\sup _{x \in K} h\left(S(x), S_{2}(x)\right) \\
\leq & \rho_{1}\left(u, u_{1}\right)+\rho_{1}\left(u, u_{2}\right)<\delta^{\prime} / 2+\delta^{\prime} / 2=\delta^{\prime} .
\end{aligned}
$$


Using (10), we have

$$
F(v) \cap\left(U_{1} \cup U_{2}\right) \neq \emptyset .
$$

If $x \in U_{1}$, then $\lambda(x)=1, \mu(x)=0, S^{\prime}(x)=S_{1}(x)$ and $\psi^{\prime}(x, y)=\psi_{1}(x, y)$ for all $y \in K$. If $x \in$ $F(v)$, then $x \in S^{\prime}(x)$ and $\psi^{\prime}(x, y) \notin-\operatorname{int} C_{n}$ for all $y \in S^{\prime}(x)$. Since $S^{\prime}(x)=S_{1}(x)$ and $\psi^{\prime}(x, y)=$ $\psi_{1}(x, y)$ for all $y \in K$, we have $x \in F\left(u_{1}\right)$. This contradicts (11). Thus, we have $F(v) \cap U_{1}=\emptyset$. Similarly, we can show that $F(v) \cap U_{2}=\emptyset$. This contradicts (12). Hence, $m(u)$ is connected, so the conclusion $(a)$ holds.

(b) For any $u \in H$, by $(a)$, there exists at least one essential connected set $m$ of $F(u)$. There exists a component $F_{\alpha}(u)$ of $F(u)$ such that $m \subset F_{\alpha}(u)$. It is obvious that $F_{\alpha}(u)$ is essential.

Now, for each $i \in I$, let $f_{i}: K \times K_{i} \rightarrow Y_{i}$ be a vector-valued mapping and $S_{i}: K \rightarrow 2^{K_{i}}$ a set-valued mapping. Let

$D=\left\{\left(f_{1}, \ldots, f_{i}, \ldots, f_{n}\right)\right.$ : for each $i \in I=\{1,2, \ldots, n\}, f_{i}(\cdot, \cdot)$ is $\left(-C_{i}\right)$-continuous on $K \times K_{i}$; for each $i \in I$ and each fixed $x \in K, f_{i}(x, \cdot)$ is $C_{i}$-convex; for each $i \in I$ and each $x \in K, f_{i}\left(x, x_{i}\right)=\theta$, where $x_{i}$ is the $i$ th component of $x$ and $\theta$ is the zero element of $Y_{i}$; for each $i \in I, \sup _{\left(x, y_{i}\right) \in K \times K_{i}}\left\|f_{i}\left(x, y_{i}\right)\right\|<+\infty$, where $\|\cdot\|$ is the norm on $\left.Y_{n}\right\}$.

Let

$Q=\left\{\left(S_{1}, \ldots, S_{i}, \ldots, S_{n}\right)\right.$ : for each $i \in I=\{1,2, \ldots, n\}$ and each $x \in K, S_{i}(x)$ is a nonempty compact convex subset of $K_{i}$; for each $i \in I, S_{i}$ is continuous on $\left.K\right\}$.

Let $P=D \times Q$. For any

$$
p_{1}=\left(\left(f_{11}, \ldots, f_{1 n}\right),\left(S_{11}, \ldots, S_{1 n}\right)\right), \quad p_{2}=\left(\left(f_{21}, \ldots, f_{2 n}\right),\left(S_{21}, \ldots, S_{2 n}\right)\right) \in P,
$$

define

$$
\rho_{2}\left(p_{1}, p_{2}\right)=\sum_{i=1}^{n} \sup _{\left(x, y_{i}\right) \in K \times K_{i}}\left\|f_{1 i}\left(x, y_{i}\right)-f_{2 i}\left(x, y_{i}\right)\right\|+\sup _{x \in K} h\left(\prod_{i=1}^{n} S_{1 i}(x), \prod_{i=1}^{n} S_{2 i}(x)\right),
$$

where $h$ is the Hausdorff metric defined on $C K(X)$. Clearly, $\left(P, \rho_{2}\right)$ is a metric space.

Let $K=\prod_{i=1}^{n} K_{i}$. It is clear that $K$ is a nonempty compact convex subset of $X=\prod_{i=1}^{n} X_{i}$. For any $\left(f_{1}, \ldots, f_{n}\right) \in D$, and $\left(S_{1}, \ldots, S_{n}\right) \in Q$, define the mapping $\psi: K \times K \rightarrow Y_{n}$ by

$$
\psi(x, y)=\sum_{i=1}^{n} f_{i}\left(x, y_{i}\right), \quad x=\left(x_{1}, \ldots, x_{n}\right), y=\left(y_{1}, \ldots, y_{i}, \ldots, y_{n}\right) \in K
$$

and the mapping $S: K \rightarrow 2^{K}$ by

$$
S(x)=\prod_{i=1}^{n} S_{i}(x), \quad x \in K
$$


Since $\left(f_{1}, \ldots, f_{n}\right) \in D$ and $\left(S_{1}, \ldots, S_{n}\right) \in Q$, we can see that $S: K \rightarrow 2^{K}$ is continuous with nonempty convex and compact valued, (i) $\psi(\cdot, \cdot)$ is $\left(-C_{n}\right)$-continuous on $K \times K$; (ii) for each fixed $x \in K, \psi(x, \cdot)$ is $\left(-C_{n}\right)$-convex; and (iii) $\psi(x, x)=\theta \notin-\operatorname{int} C_{n}$ for all $x \in K$. It is clear that $(\psi, S) \in H$. Since $Y_{i} \subset Y_{n}$ for each $i \in I=\{1,2, \ldots, n\}$, by Theorem 2.1, there exists an element $x^{*} \in K$ such that $x^{*} \in S\left(x^{*}\right)$ and

$$
\psi\left(x^{*}, y\right) \notin-\operatorname{int} C_{n} \quad \text { for all } y \in S\left(x^{*}\right) .
$$

That is

$$
f_{1}\left(x^{*}, y_{1}\right)+\cdots+f_{i}\left(x^{*}, y_{i}\right)+\cdots+f_{n}\left(x^{*}, y_{n}\right) \notin-\operatorname{int} C_{n} \quad \text { for all } y_{1} \in S_{1}\left(x^{*}\right), \ldots, y_{n} \in S_{n}\left(x^{*}\right) \text {. }
$$

For each $i \in I$, by the arbitrariness of $y_{j} \in S_{j}\left(x^{*}\right), j \in\{1, \ldots, n\}, j \neq i$, take $y_{j}=x_{j}^{*}$, and by assumption $f_{j}\left(x^{*}, x_{j}^{*}\right)=\theta, j=1, \ldots, n$, and $j \neq i$, we obtain that $x_{i}^{*} \in S_{i}\left(x^{*}\right)$ and

$$
f_{i}\left(x^{*}, y_{i}\right) \notin-\operatorname{int} C_{n} \quad \text { for all } y_{i} \in S_{i}\left(x^{*}\right) \text {. }
$$

Since $f_{i}\left(x^{*}, y_{i}\right) \in Y_{i}$ and $C_{i}=C_{n} \cap Y_{i}$, it follows that

$$
f_{i}\left(x^{*}, y_{i}\right) \notin-\operatorname{int} C_{i} \quad \text { for all } y_{i} \in S_{i}\left(x^{*}\right) \text {. }
$$

Thus, there exists $x^{*}=\left(x_{1}^{*}, \ldots, x_{n}^{*}\right) \in K$ such that for each $i \in I, x_{i}^{*} \in S_{i}\left(x^{*}\right)$ and

$$
f_{i}\left(x^{*}, y_{i}\right) \notin-\operatorname{int} C_{i} \quad \text { for all } y_{i} \in S_{i}\left(x^{*}\right) \text {. }
$$

For each $p \in P$, denote by $E(p)$ all solutions to the SVQEP. By (13), there exists $x^{*} \in E(p)$, thus $E(p) \neq \emptyset$. Similar to Definition 3.1, we can define the minimal essential set and essential component of $E(p)$.

Lemma 3.4 For each $p=\left(\left(f_{1}, \ldots, f_{n}\right),\left(S_{1}, \ldots, S_{n}\right)\right) \in P$, define the mapping $T: P \rightarrow H$ by

$$
T(p)=(\psi, S),
$$

where

$$
\psi(x, y)=\sum_{i=1}^{n} f_{i}\left(x, y_{i}\right), \quad x=\left(x_{1}, \ldots, x_{n}\right), y=\left(y_{1}, \ldots, y_{i}, \ldots, y_{n}\right) \in K
$$

and

$$
S(x)=\prod_{i=1}^{n} S_{i}(x), \quad x \in K .
$$

Then $T$ is continuous.

Proof It is easy to check that for each $p=\left(\left(f_{1}, \ldots, f_{n}\right),\left(S_{1}, \ldots, S_{n}\right)\right) \in P, T(p)=(\psi, S) \in H$. 
For any $p_{1}=\left(\left(f_{11}, \ldots, f_{1 n}\right),\left(S_{11}, \ldots, S_{1 n}\right)\right), \quad p_{2}=\left(\left(f_{21}, \ldots, f_{2 n}\right),\left(S_{21}, \ldots, S_{2 n}\right)\right) \in P$, if $\rho_{2}\left(p_{1}, p_{2}\right)<\varepsilon$, then by the definition of $\rho_{1}$, we have

$$
\begin{aligned}
\rho_{1}( & \left.T\left(p_{1}\right), T\left(p_{2}\right)\right) \\
= & \sup _{(x, y) \in K \times K}\left\|\sum_{i=1}^{n} f_{1 i}\left(x, y_{i}\right)-\sum_{i=1}^{n} f_{2 i}\left(x, y_{i}\right)\right\| \\
& \quad+\sup _{x \in K} h\left(\prod_{i=1}^{n} S_{1 i}(x), \prod_{i=1}^{n} S_{2 i}(x)\right) \leq \sup _{(x, y) \in K \times K}\left\|f_{11}\left(x, y_{1}\right)-f_{21}\left(x, y_{1}\right)\right\| \\
& +\cdots+\sup _{(x, y) \in K \times K}\left\|f_{1 n}\left(x, y_{n}\right)-f_{2 n}\left(x, y_{n}\right)\right\|+\sup _{x \in K} h\left(\prod_{i=1}^{n} S_{1 i}(x), \prod_{i=1}^{n} S_{2 i}(x)\right) \\
= & \sum_{i=1}^{n} \sup _{\left(x, y_{i}\right) \in K \times K_{i}}\left\|f_{1 i}\left(x, y_{i}\right)-f_{2 i}\left(x, y_{i}\right)\right\|+\sup _{x \in K} h\left(\prod_{i=1}^{n} S_{1 i}(x), \prod_{i=1}^{n} S_{2 i}(x)\right) \\
\leq & \rho_{2}\left(p_{1}, p_{2}\right)<\varepsilon .
\end{aligned}
$$

This completes the proof of the lemma.

The following lemma can be found in [8].

Lemma 3.5 Let $U, Y$ and $Z$ be three metric spaces, $F: U \rightarrow 2^{Y}$ be an usco mapping and $G: Z \rightarrow 2^{Y}$ be a set-valued mapping. Suppose that there exists a continuous mapping $T$ : $Z \rightarrow U$ such that $G(z) \supset F(T(z))$ for each $z \in Z$. Furthermore, suppose that there exists at least one essential component of $F(\varphi)$ for each $\varphi \in U$. Then there exists at least one essential component of $G(z)$ for each $z \in Z$.

As application of Theorem 3.1, now we will show that, for every system of vector quasiequilibrium problems, there exists at least one essential component of the set of its solutions in the uniform topological space of objective functions and constraint mappings.

Theorem 3.2 For each $p \in P$, there exists at least one essential component of $E(p)$.

Proof For any $p=\left(\left(f_{1}, \ldots, f_{n}\right),\left(S_{1}, \ldots, S_{n}\right)\right) \in P$, define $T: P \rightarrow H$ by $T(p)=(\psi, S)$, where

$$
\psi(x, y)=\sum_{i=1}^{n} f_{i}\left(x, y_{i}\right), \quad x=\left(x_{1}, \ldots, x_{n}\right), y=\left(y_{1}, \ldots, y_{i}, \ldots, y_{n}\right) \in K
$$

and

$$
S(x)=\prod_{i=1}^{n} S_{i}(x), \quad x \in K .
$$

By Lemma 3.4, $T$ is continuous. Now we need to prove that for each $p \in P, E(p) \supset F(T(p))$, where $F$ is defined by (1). If $x^{*}=\left(x_{1}^{*}, \ldots, x_{i}^{*}, \ldots, x_{n}^{*}\right) \in F(T(p))$, then $x^{*} \in K, x^{*} \in S\left(x^{*}\right)$ and

$$
\psi\left(x^{*}, y\right) \notin-\operatorname{int} C_{n} \quad \text { for all } y \in S\left(x^{*}\right) \text {. }
$$


That is

$$
f_{1}\left(x^{*}, y_{1}\right)+\cdots+f_{i}\left(x^{*}, y_{i}\right)+\cdots+f_{n}\left(x^{*}, y_{n}\right) \notin-\operatorname{int} C_{n} \quad \text { for all } y_{1} \in S_{1}\left(x^{*}\right), \ldots, y_{n} \in S_{n}\left(x^{*}\right) \text {. }
$$

For each $i \in I$, by the arbitrariness of $y_{j} \in S_{j}\left(x^{*}\right), j \in\{1, \ldots, n\}, j \neq i$, take $y_{j}=x_{j}^{*}$, and by assumption $f_{j}\left(x^{*}, x_{j}^{*}\right)=\theta, j=1, \ldots, n$, and $j \neq i$, we obtain that $x_{i}^{*} \in S_{i}\left(x^{*}\right)$ and

$$
f_{i}\left(x^{*}, y_{i}\right) \notin-\operatorname{int} C_{n} \quad \text { for all } y_{i} \in S_{i}\left(x^{*}\right) \text {. }
$$

Since $f_{i}\left(x^{*}, y_{i}\right) \in Y_{i}$ and $C_{i}=C_{n} \cap Y_{i}$, it follows that

$$
f_{i}\left(x^{*}, y_{i}\right) \notin-\operatorname{int} C_{i} \quad \text { for all } y_{i} \in S_{i}\left(x^{*}\right) \text {. }
$$

Hence $x^{*} \in E(p)$ and hence $E(p) \supset F(T(p))$. Thus, by Lemma 3.2, Theorem 3.1 and Lemma 3.5, there exists at least one essential component of $E(p)$.

\section{Competing interests}

The authors declare that they have no competing interests.

\section{Authors' contributions}

This work was carried out in collaboration between all authors. X-HG gave the ideas of the problems in this research and interpreted the results. J-CC proved the theorems, interpreted the results and wrote the article. All authors defined the research theme, read and approved the manuscript.

\section{Author details}

${ }^{1}$ Department of Mathematics, Nanchang University, Nanchang, 330031, China. ${ }^{2}$ College of Mathematics and Information Science, Nanchang Hangkong University, Nanchang, 330063, China.

\section{Acknowledgements}

This research was partially supported by the National Natural Science Foundation of China (Grant No. 11061023) and the Natural Science Foundation of Jiangxi Province (2010GZS0176), China.

Received: 11 May 2012 Accepted: 3 August 2012 Published: 30 August 2012

\section{References}

1. Fort, MK: Essential and nonessential fixed points. Am. J. Math. 72, 315-322 (1950)

2. Kinoshita, S: On essential component of the set of fixed points. Osaka J. Math. 4, 19-22 (1952)

3. Jiang, $\mathrm{JH}$ : Essential components of the set of fixed points of multivalued mappings and its application to the theory of games. Sci. Sin. 12, 951-964 (1963)

4. Kohlberg, E, Mertens, JF: On the strategic stability of equilibria. Econometrica 54, 1003-1037 (1986)

5. Yu, J, Xiang, SW: On essential components of the set of Nash equilibrium points. Nonlinear Anal., Theory Methods Appl. 38, 259-264 (1999)

6. Yu, J, Luo, Q: On essential components of the solution set of generalized games. J. Math. Anal. Appl. 230, 303-310 (1999)

7. Isac, G, Yuan, GXZ: The essential components of coincident points for weakly inward and outward set-valued mappings. Appl. Math. Lett. 12, 121-126 (1999)

8. Yang, $H, Y u$, J: Essential components of the set of weakly Pareto-Nash equilibrium points. Appl. Math. Lett. 15 553-560 (2002)

9. Lin, Z: Essential components of the set of weakly Pareto-Nash equilibrium points for multiobjective generalized games in two different topological spaces. J. Optim. Theory Appl. 124, 387-405 (2005)

10. Chen, JC, Gong, XH: The stability of set of solutions for symmetric vector quasi-equilibrium problems. J. Optim. Theory Appl. 136, 359-374 (2008)

11. Ansari, QH, Schaible, S, Yao, JC: The system of generalized vector equilibrium problems with applications. J. Glob. Optim. 22, 3-16 (2002)

12. Ansari, QH, Chan, WK, Yang, XQ: The system of vector quasi-equilibrium problems with applications. J. Glob. Optim. 29, 45-57 (2004)

13. Fang, YP, Huang, NJ, Kim, JK: Existence results for system of vector equilibrium problems. J. Glob. Optim. 35, 71-83 (2006)

14. Peng, JW, Lee, HWJ, Yang, XM: On system of generalized vector quasi-equilibrium problems with set-valued maps. J. Glob. Optim. 36, 139-158 (2006)

15. Lin, Z: The study of the system of generalized vector quasi-equilibrium problems. J. Glob. Optim. 36, 627-635 (2006) 
16. Peng, JW, Yang, XM, Zhu, DL: System of vector quasi-equilibrium and its applications. Appl. Math. Mech. 27, 1107-1114 (2006)

17. Chen, JC, Gong, XH: Generic stability of the solution set for symmetric vector quasi-equilibrium problems under the condition of cone-convexity. Acta Math. Sci. 30, 1006-1017 (2010)

18. Yang, $\mathrm{H}, \mathrm{Yu}, \mathrm{J}$ : The generic stability and the existence of essential components of the solution sets for generalized vector variational-like inequalities. J. Syst. Sci. Math. Sci. 22, 90-95 (2002) (in Chinese)

19. Aubin, JP, Ekeland, I: Applied Nonlinear Analysis. Wiley, New York (1984)

20. Engelking, R: General Topology. Helderman, Berlin (1989)

doi:10.1186/1029-242X-2012-181

Cite this article as: Gong and Chen: Essential components of the set of solutions for the system of vector quasi-equilibrium problems. Journal of Inequalities and Applications 2012 2012:181.

Submit your manuscript to a SpringerOpen ${ }^{\circ}$ journal and benefit from:

- Convenient online submission

- Rigorous peer review

Immediate publication on acceptance

- Open access: articles freely available online

- High visibility within the field

- Retaining the copyright to your article 